\title{
NMR STUDY OF ION MOTIONS IN $\left[\mathrm{C}\left(\mathrm{NH}_{2}\right)_{3}\right]_{3} \mathrm{GaF}_{6}$
}

\author{
A. KozAK ${ }^{a}$, M. GRotTeL ${ }^{b}$ AND Z. PAJĄK ${ }^{a}$ \\ ${ }^{a}$ Institute of Physics, A. Mickiewicz University, Poznan, Poland \\ ${ }^{b}$ Department of Physics, Academy of Agriculture, Poznań, Poland
}

(Received October 1, 1992)

\begin{abstract}
Proton and fluorine spin-lattice relaxation times as well as second moments were measured in guanidinium hexafluorogallate over a wide range of temperature. The analysis of all cross-relaxation effects occurring in the four unlike spin system revealed two cation motions and yielded their activation parameters. It was found that the anion size did not influence $C_{3}$ reorientation of cation nor isotropic reorientation of anion.
\end{abstract}

PACS numbers: 76.60.-k, 76.60.Es

\section{Introduction}

In our previous studies of various guanidinium salts a molecular dynamics of cation embedded in different anion sublattices was analyzed [1-5]. It was particularly interesting to examine a role of the cation-anion interactions and hydrogen bondings contributing essentially to potential barrier which hinders the considered cation reorientation. In all the compounds studied we found an evident existence of the reorientation of the whole guanidinium cation around its $C_{3}$ symmetry axis. The activation energies found for this reorientation vary from $26 \mathrm{~kJ} / \mathrm{mol}$ for weak bonds up to $68 \mathrm{~kJ} / \mathrm{mol}$ for strong hydrogen bonds. In our study of the guanidinium complexes containing fluorinated anions it was possible to describe dynamics of both ionic sublattices and reveal an interesting interaction of cations and anions leading to a coupling of the reorientational frequencies at phase transition [6]. In most compounds the onset of cation motion requires much higher energy as compared with that required for the anion motion. We have recently found the only exceptional behaviour in the guanidinium hexafluoroaluminate [5] wherein activation energy for the anion motion $(67 \mathrm{~kJ} / \mathrm{mol})$ evidently exceeded that found for the cation $(45 \mathrm{~kJ} / \mathrm{mol})$. Hence the relative rigidity of the anion sublattice in the wide temperature range enabled us to confirm an existence of another cation motion, suggested earlier in our previous works.

It seemed interesting to examine the hexafluorogallate, the compound which, besides hexafluoroindate, is isostructural with the guanidinium hexafluoroaluminate [7]. No essential difference in crystal and molecular structure has been observed in both compounds $[5,8]$. The hexafluorogallate crystallizes in a cubic 
system, in the space group $P 2_{1} / a \overline{3}, a=14.073(1) \AA, V=2787.1 \AA^{3}$ and $Z=8$. The molecular packing can be described as a framework of $\mathrm{GaF}_{6}$ ions, hydrogen bonded to the guanidinium cations.

In the present work a study of ${ }^{1} \mathrm{H}$ and ${ }^{19} \mathrm{~F}$ NMR second moment and spin-lattice relaxation time $T_{1}$ was undertaken to obtain information on the dynamics of both sublattices in the compound and compare it with those for the guanidinium hexafluoroaluminate. It seemed interesting to find out whether a size of an anion influences a height of the barrier hindering reorientations of both ions and to confirm an existence of a complex motion of the cation.

\section{Experimental}

The guanidinium hexafluorogallate $\left[\mathrm{C}\left(\mathrm{NH}_{2}\right)_{3}\right]_{3} \mathrm{GaF}_{6}$ was obtained following the reaction:

$$
\begin{aligned}
& 3\left[\mathrm{C}\left(\mathrm{NH}_{2}\right)_{3}\right]_{2} \mathrm{CO}_{3}+\mathrm{Ga}_{2} \mathrm{O}_{3}+12 \mathrm{HF} \longrightarrow \\
& \longrightarrow 2\left[\mathrm{C}\left(\mathrm{NH}_{2}\right)_{3}\right]_{3} \mathrm{GaF}_{6}+3 \mathrm{CO}_{2}+6 \mathrm{H}_{2} \mathrm{O} .
\end{aligned}
$$

Respective amounts of guanidinium carbonate and gallium oxide were treated with an excess of hydrofluoric acid HF ( $40 \%$ aqueous solution) and heated to a complete dissolution. After cooling small cubes of $\left[\mathrm{C}\left(\mathrm{NH}_{2}\right)_{3}\right]_{3} \mathrm{GaF}_{6}$ crystallized from the solution. Analysis - found: $\mathrm{N}=34.65, \mathrm{C}=9.91, \mathrm{H}=4.73 \%$; calculated: $\mathrm{N}=34.64, \mathrm{C}=9.90, \mathrm{H}=4.98 \%$. To remove iron paramagnetic impurities, the compound was then recrystallized from $15 \%$ HF solution with a small amount of tartaric acid added. The product was then ground to a powder, dried, degassed and sealed off.

Measurements of proton and fluorine second moment were done over a wide range of temperature with our NMR spectrometer operating at the Larmor frequency of 28.0 and $26.3 \mathrm{MHz}$, for protons and fluorines, respectively. Measurements of the proton and fluorine spin-lattice relaxation times $T_{1}$ were performed in a function of temperature using a home-made pulse spectrometer operating at $40 \mathrm{MHz}$ by a $\pi / 2-\tau-\pi / 2$ pulse sequence.

Temperature of the sample was controlled by means of a gas-flow cryostat and monitored with a $\mathrm{Pt}$ resistor to an accuracy of about $1 \mathrm{~K}$. Differential thermal analysis was made with a Derivatograph UNIPAN (type DSC 605M).

\section{Results}

Experimental proton and fluorine NMR second moments in a function of temperature are presented in Fig. 1 . The proton second moment of about $26 \mathrm{G}^{2}$ starts to decrease slowly at about $150 \mathrm{~K}$ to a value of $24 \mathrm{G}^{2}$ and then at $270 \mathrm{~K}$ sharply to a value of $5.2 \mathrm{G}^{2}$ achieved above $420 \mathrm{~K}$. The fluorine second moment decreases from the value of $16.1 \mathrm{G}^{2}$ registered at low temperatures to a plateau value of about $7.5 \mathrm{G}^{2}$ at temperature range $350 \mathrm{~K}-440 \mathrm{~K}$. At higher temperature the fluorine second moment further diminishes to a value of $1.4 \mathrm{G}^{2}$ registered at $530 \mathrm{~K}$. The full curves in Fig. 1 are theoretically calculated for assumed model of the cation reorientation. 


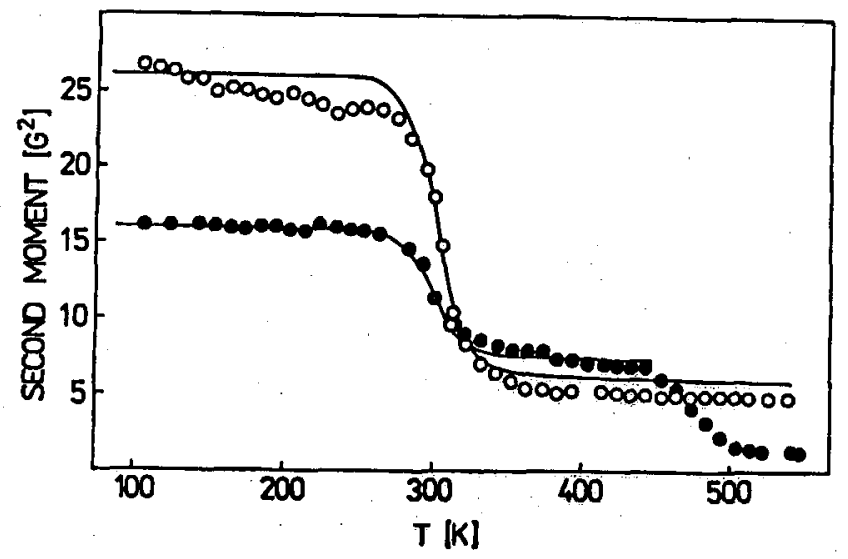

Fig. 1. Temperature dependencies of ${ }^{1} H(0)$ and ${ }^{19} \mathrm{~F}(\bullet)$ NMR second moments. Full curves are theoretically calculated for the $C_{3}$ reorientation of the guanidinium cation.

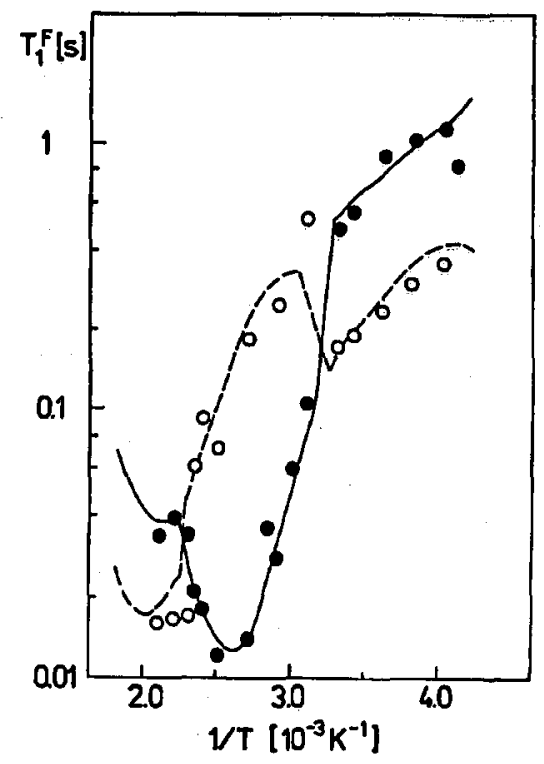

Fig. 2. Temperature dependence of the fluorine spin-lat tice relaxation time: $\bullet-$ components for magnetization amplitudes higher than 0.5 ; solid and dashed lines denote calculated $\lambda_{\mathrm{F}}^{-1}$ and $\lambda_{\mathrm{H}}^{-1}$, respectively.

Figure 2 presents the fluorine spin-lattice relaxation time as $\log T_{1}^{\mathrm{F}}$ plot against inverse temperature. The curves in the figure are theoretical fits to the experimental data. At all the temperatures studied a non-exponential magnetization decay was observed. The decay curves were decomposed into two exponential 
terms with different $T_{1}$ values. In Fig. 2 the full circles denote $T_{1}^{\mathrm{F}}$ components for fluorine magnetization amplitudes higher than 0.5. One can see an evident minimum of $12 \mathrm{~ms}$ at about $385 \mathrm{~K}$ revealed in the plot of the short components.

Differential thermal analysis performed from room temperature to $600 \mathrm{~K}$ revealed that the compound started to decompose at about $560 \mathrm{~K}$.

\section{Calculations and discussion}

\subsection{NMR second moment}

Experimental proton and fluorine second moments obtained in a function of temperature remind those found for $\left[\mathrm{C}\left(\mathrm{NH}_{2}\right)_{3}\right]_{3} \mathrm{AlF}_{6}[5]$. The lower value of the fluorine second moment $\left(16.1 \mathrm{G}^{2}\right)$ found for $\left[\mathrm{C}\left(\mathrm{NH}_{2}\right)_{3}\right]_{3} \mathrm{GaF}_{6}$ (see Fig. 1) comes from the smaller intramolecular F-F $\left(3.48 \mathrm{G}^{2}\right)$ and F-Ga $\left(1.20 \mathrm{G}^{2}\right)$ contributions evidently resulting from the $\mathrm{Ga}-\mathrm{F}$ distance $(1.901 \AA)$ longer than $\mathrm{Al}-\mathrm{F}$ length $(1.818 \AA)$. Hence, the theoretical fluorine second moment of $16.1 \mathrm{G}^{2}$ is exactly the same as experimental value $\left(16.1 \mathrm{G}^{2}\right)$, thus proving the rigidity of the $\mathrm{GaF}_{6}$ anion sublattice at low temperatures. Therefore, it follows that interionic fluorine-proton and proton-fluorine contributions to the respective second moments must be the same as for the isostructural $\left[\mathrm{C}\left(\mathrm{NH}_{2}\right)_{3}\right]_{3} \mathrm{AlF}_{6}$. Hence the theoretical proton second moment value of $21.6 \mathrm{G}^{2}$ found for the aluminate can be accepted as corresponding to the rigid cation sublattice in the gallate, as well. The reduction of the proton and fluorine second moments observed upon increase in temperature can be interpreted similarly as in case of the aluminium analogue. The one-step reduction of the proton second moment can be ascribed to the reorientation of the guanidinium cation around its $C_{3}$ symmetry axis perpendicular to the cation plane. Theoretical plot calculated using the Bloembergen-Purcell-Pound formula [9] and activation parameters extracted from our relaxation data compared with the experiment evidently shows that there must exist another motion which precedes the $C_{3}$ reorientation and further reduces the second moment value at high temperatures. It is not clear what kind of motion it is and why it does not affect the measured fluorine second moment at low temperatures. It can be a reorientation of $\mathrm{NH}_{2}$ groups or small angle over the barrier jumps of the whole cation. The respective theoretical plot calculated for fluorines proves that the plateau value observed for the fluorine second moment at temperature range $350-440 \mathrm{~K}$ reflects only the onset of the $C_{3}$ reorientation of the guanidinium cation and cannot be explained by any reorientation of the anion. The latter starts to reorient as late as at about $440 \mathrm{~K}$. The value of $1.4 \mathrm{G}^{2}$ registered at the highest temperatures indicates that it is an isotropic reorientation hindered by a high potential barrier of about $68 \mathrm{~kJ} / \mathrm{mol}$, as estimated from the Waugh-Fiedin formula [10]. This value is comparable with that found for the $\mathrm{AlF}_{6}$ anions. Since the barrier for the anion tumbling is of the interionic origin, the fluorine-proton interactions must be the same in both compounds. 


\section{2. $N M R$ relaxation}

To describe our experimental proton and fluorine relaxation data we had to consider all homo- and heteronuclear interactions occurring in the compound studied. Since it is a system of four unlike spins (H, F, N, and Ga), we could apply the solution of a set of coupled differential equations describing the time variation of nuclear magnetization derived in [4]:

$$
\left[\begin{array}{c}
\mathrm{d} M_{\mathrm{H}} / \mathrm{d} t \\
\mathrm{~d} M_{\mathrm{F}} / \mathrm{d} t \\
\mathrm{~d} M_{\mathrm{N}} / \mathrm{d} t \\
\mathrm{~d} M_{\mathrm{Ga}} / \mathrm{d} t
\end{array}\right]=\left[\begin{array}{cccc}
-R_{\mathrm{HH}} & -R_{\mathrm{HF}} & -R_{\mathrm{HN}} & -R_{\mathrm{HGa}} \\
-R_{\mathrm{FH}} & -R_{\mathrm{FF}} & -R_{\mathrm{FN}} & -R_{\mathrm{FGa}} \\
-R_{\mathrm{NH}} & -R_{\mathrm{NF}} & -R_{\mathrm{NN}} & -R_{\mathrm{NGa}} \\
-R_{\mathrm{GaH}} & -R_{\mathrm{GaF}} & -R_{\mathrm{GaN}} & -R_{\mathrm{GaGa}}
\end{array}\right]\left[\begin{array}{c}
M_{\mathrm{H}}-M_{0 \mathrm{H}} \\
M_{\mathrm{F}}-M_{0 \mathrm{~F}} \\
M_{\mathrm{N}}-M_{0 \mathrm{~N}} \\
M_{\mathrm{Ga}}-M_{0 \mathrm{Ga}}
\end{array}\right]
$$

To find the eigenvalues of the relaxation matrix and compare them with the experimental proton and/or fluorine values one must estimate all homo- and heteronuclear contributions to the reduced second moments for the assumed model of ion reorientation and calculate all diagonal $\left(R_{I I}\right)$ and off-diagonal $\left(R_{I S}\right)$ elements according to the formulae:

$$
\begin{aligned}
& R_{I I}=(2 / 3) \gamma_{I}^{2} \Delta M_{2}^{I I} g_{1}\left(\omega_{I}, \tau\right)+(1 / 2) \sum_{S} \gamma_{I}^{2} \Delta M_{2}^{I S} g_{2}\left(\omega_{I}, \omega_{S}, \tau\right), \\
& R_{I S}=(1 / 2) \gamma_{S}^{2} \Delta M_{2}^{S I} g_{3}\left(\omega_{I}, \omega_{S}, \tau\right) N_{S} / N_{I},
\end{aligned}
$$

where

$$
\begin{aligned}
& g_{1}\left(\omega_{I}, \tau\right)=\tau /\left(1+\omega_{I}^{2} \tau^{2}\right)+4 \tau /\left(1+4 \omega_{I}^{2} \tau^{2}\right), \\
& g_{2}\left(\omega_{I}, \omega_{S}, \tau\right)=\tau /\left[1+\left(\omega_{I}-\omega_{S}\right)^{2} \tau^{2}\right]+3 \tau /\left(1+\omega_{I}^{2} \tau^{2}\right) \\
& \left.+6 \tau /\left[1+\omega_{I}+\omega_{S}\right)^{2} \tau^{2}\right], \\
& g_{3}\left(\omega_{I}, \omega_{S}, \tau\right)=-\tau /\left[1+\left(\omega_{I}-\omega_{S}\right)^{2} \tau^{2}\right]+6 \tau /\left[1+\left(\omega_{I}+\omega_{S}\right)^{2} \tau^{2}\right] .
\end{aligned}
$$

As it results from our second moment study, we have considered the $C_{3}$ rcorientation of the guanidinium cation preceded by the other motion while the anions are still rigid. Hence we considered the relaxation matrix as a sum of two components

$$
R=R_{1}+R_{2}
$$

corresponding to both motions. Then, we calculated eigenvalues $\lambda_{\mathbf{r}}$ of the relaxation matrix and their respective magnetization amplitudes $A(r)$ in a function of tcmperature. The inverses of the eigenvalues $\lambda_{\mathrm{H}}^{-1}$ and $\lambda_{\mathrm{F}}^{-1}$ were then compared with the respective experimental short and long components of the $T_{1}$ values.

For the fluorine experiment (see Fig. 2) a good agreement is observed at temperatures where only fluorine-proton interaction contributes to the fluorine magnetization. It turned out that in the whole temperature range the nitrogen and gallium contributions to the fluorine magnetization are negligibly small and can be neglected. Hence our compound can be treated as a two-spin system and a decomposition of fluorine magnetization recovery into two exponential decays is fully justified. Thus, the fluorine experiment appears to be sufficient to reveal the 
molecular dynamics of the cation and confirms an existence of two motions. The relaxation at temperatures higher than $320 \mathrm{~K}$ is mainly due to the $C_{3}$ reorientation of the cation. The minimum observed at about $385 \mathrm{~K}$ is related to this reorientation through the heteronuclear proton-fluorine interaction $\tau \approx\left(\omega_{\mathrm{H}}-\omega_{\mathrm{F}}\right)^{-1}$. A significant shortening of the fluorine $T_{1}$ values at temperatures lower than $320 \mathrm{~K}$ and the inversion of the magnetization amplitudes must result from an existence of another cation motion, which precedes its $C_{3}$ reorientation. The best fitting of the theoretical eigenvalues $\lambda_{\mathrm{H}}^{-1}$ and $\lambda_{\mathrm{F}}^{-1}$ to the experimental $T_{1}^{\mathrm{F}}$ data yielded the Arrhenius activation parameters for the two motions considered (Table).

\section{TABLE}

Activation parameters for the cation motions.

\begin{tabular}{c|c|c}
\hline \hline & $E_{\mathrm{a}}[\mathrm{kJ} / \mathrm{mol}]$ & $\tau_{0}[\mathrm{~s}]$ \\
\hline$C_{3}$ reorientation & 44.4 & $5.94 \times 10^{-14}$ \\
The other motion & 18.8 & $2.01 \times 10^{-12}$
\end{tabular}

From the fitting procedure it turns out that the motion preceding the $C_{3}$ reorientation only very slightly modulates the fluorine-proton interaction $\left(\Delta M_{2}(\mathrm{~F}-\mathrm{H})=0.2 \mathrm{G}^{2}\right)$. Therefore, it is not surprising that this motion does not affect, in the accuracy limit, the fluorine second moment at low temperatures. The geometry of the motion cannot be precisely defined, since an X-ray analysis does not reveal exact hydrogen atom positions [11].

For protons an agreement between the calculated eigenvalues and experimental components of $T_{1}^{\mathrm{H}}$ is somewhat worse due to more complicated heteronuclear interactions (nitrogen and gallium contributions cannot be neglected). However, the proton relaxation data also reveal and corroborate the model of cation reorientations derived from the fluorine experiment.

Our analysis shows that molecular dynamics of both sublattices in the compound studied is very similar to that of the guanidinium hexafuoroaluminate. The cation's freedom to reorient is much higher than the anion's one. It results from hydrogen bond pattern: each anion is involved in three times more hydrogen bonds than the cation. The relative rigidity of the anion sublattice in a wide temperature range allowed us to confirm once again the existence of the additional motion of the cation preceding its $C_{3}$ reorientation. Though the activation energies for the $C_{3}$ reorientation of cation and isotropic reorientation of anion are the same in the gallate and in the aluminate, it is not clear why the hindering barrier for the additional motion of the cation is evidently lower in the gallate. It could suggest its intraionic origin, resulting maybe from the tiny difference in the molecular structure of the cations in both salts. Hence one can suppose that the additional motion can be rather related to the $\mathrm{NH}_{2}$ groups than to the whole cation. Since the crystal structure of both salts is nearly the same, a significant increase in the $\mathrm{GaF}_{6}$ anion size compared with $\mathrm{AlF}_{6}$, revealed in the $\mathrm{X}$-ray analysis, docs not 
essentially affect the height of the barriers hindering the $C_{3}$ reorientation of the cation nor the isotropic reorientation of the anion.

\section{Acknowledgment}

The present work has been supported by the Committee for Scientific Research under Grant No. 1427/2/91. ments.

The authors wish to thank Dr. S. Lewicki for his assistance in $T_{1}$ measure-

\section{References}

[1] Z. Pajak, M. Grottel, A.E. Koziol, J. Chem. Soc. Faraday Trans. 2 78, 1529 (1982).

[2] M. Grottel, Z. Pajak, J. Chem. Soc. Faraday Trans. 2 80, 553 (1984).

[3] A. Kozak, M. Grottel, A.E. Koziol, Z. Pajak, J. Phys. C, Solid State Phys. 20, 5433 (1987).

[4] M. Grottel, A. Kozak, A.E. Koziol, Z. Pajak, J. Phys., Condens. Matter 1, 7069 (1989).

[5] M. Grottel, A. Kozak, H. Małuszyńska, Z. Pająk, J. Phys., Condens. Matter 4, 1837 (1992).

[6] Z. Pajqk, A. Kozak, M. Grottel, Solid State Commun. 65, 671 (1988).

[7] P. Bukovec, Monatsh. Chem. 114, 277 (1983).

[8] H. Małuszyńska, Acta Crystallogr. C, submitted for publication.

[9] N. Bloembergen, E.M. Purcell, R.V. Pound, Phys. Rev. 73, 679 (1948).

[10] J.S. Waugh, E.I. Fiedin, Fiz. Tverd. Tela 4, 2233 (1962).

[11] P. Schuster, G. Zundel, C. Sandorfy, The Hydrogen Bond, North-Holland, Amsterdam 1976. 